Journal of Mathematics and Informatics

Vol. 18, 2020, 85-103

ISSN: 2349-0632 (P), 2349-0640 (online)

Published 17 February 2020

www.researchmathsci.org

DOI: http://dx.doi.org/10.22457/jmi.v18a8164

Journal of

Mathematics and

Informatics

\title{
MHD Arterial Blood Flow and Mass Transfer under the \\ Presence of Stenosis, Body Acceleration and Chemical Reaction: A Case of Magnetic Therapy
}

\author{
Annord Mwapinga $^{1}$, Eunice Mureithi ${ }^{2}$,James Makung $u^{2}$ and Verdiana Masanja ${ }^{1}$ \\ ${ }^{1}$ School of Computational and Communication Science and Engineering \\ The Nelson Mandela African Institution of Science and Technology \\ P.O.Box 447, Arusha, Tanzania \\ Email: mwapingaa@nm-aist.ac.tz; verdiana.masanja@nm-aist.ac.tz \\ ${ }^{2}$ Department of Mathematics, University of Dar es Salaam \\ P.O. Box 35091 Dar es Salaam \\ Email: ewambui02@gmail.com; makungu_j@yahoo.com \\ ${ }^{1}$ Corresponding author. Email: mwapingaa@nm-aist.ac.tz
}

Received 20 January 2020; accepted 11 February 2020

\begin{abstract}
A mathematical model has been developed and used to study pulsatile blood flow and mass transfer through a stenosed artery in the presence of body acceleration and magnetic fields. An explicit Finite Difference Method (FDM) has been used to discretize the formulated mathematical model. The discretized model equations were solved in MATLAB software to produce simulations. The effect of Hartman number, Reynolds number, Schmidt number, stenotic height, body acceleration and chemical reactions have been investigated. It has been observed that, the velocity, concentration and skin friction, decrease with increasing stenotic height. Velocity on the other hand increases, as body acceleration increases. It has further been observed that as the Hartman number increases, both the radial and axial velocities diminish. Increase of the Reynolds number results in the increase of the velocity profiles. The higher the chemical reaction parameter is, the lower are the concentration profiles.
\end{abstract}

Keywords: Pulsatile flow, stenosis, body acceleration, magnetic fields, chemical reaction, magnetic therapy

AMS Mathematics Subject Classification (2010): 78A30

\section{Introduction}

The use of magnetic fields in health-related interventions is manifested in various situations, this includes in treatment of ailments. In sports such as football and athletics,magnets are used to perform magnetic therapy so as to maintain health and treat illnesses.Magnetic therapy is an alternative medical practice that uses magnets to alleviate pain and other health concerns. It is therefore possible that the magnetic therapy 
Annord Mwapinga, Eunice Mureithi, James Makungu and Verdiana Masanja

in sports can be applied to a person with stenosis because all people are susceptible to have stenosis or plaques in the body.

Biologically blood has a number of functions that are central for the survival of human being, this includes inta alia, supplying oxygen to cells and tissues, providing essential nutrients to cells (such as amino acids, fatty acids, and glucose), removing waste materials, such as carbon dioxide, urea, and lactic acid, transporting hormones from one part of the body to another. In this regard therefore, blood can be described as the transporting agent in the human body. Blood consists of red blood cells which are negatively charged. It is therefore possible that the flow of blood can get affected by the magnetic fields.

Stenosis is one of the causes of the anomalies of blood flow in arteries. The abnormal growth of deposits such as fats along the arterial wall causes reduction of the diameter of the artery and thus disturbs the normal flow of blood. In day to day activities, human being may also be subjected to external body acceleration. This includes, travelling in vehicles, airplanes, sports and other activities such as using the lathe machine or jack hammer.In this regard therefore, magnetic therapy in sports implies that, there is existence of magnetic fields and body acceleration.

Mathematical modelling of blood flow in a stenosed artery under the presence of magnetic fields has been worked on by several researchers. Kumari et al, (2019) [1], Haghighi and Aliashrafi (2018) [3], Sharma et al (2019) [4], Rajashekhar et al (2017) [7], Mwanthi et al (2017) [8], Shit et al (2014) [6], studied Magnetohydrodynamics through a stenosed artery. Their studies revealed that the application of the magnetic field causes a decrease in axial speed of blood.

Karthikeyan and Jeevitha (2019) [2], analyzed the heat and mass transfer effects on the two-phase model of the unsteady pulsatile blood flow when it flows through a stenosed artery with permeable wall under the effect of chemical reaction. The study showed that as the chemical reaction parameter increases, the concentration profiles decrease.Further, plots of the volumetric flow rate and the velocity exhibitsinusoidal behavior with time. A similar study with similar results was done by Hossain and Haque (2017) [13] for bifurcated artery. These studies neglected the presence of body acceleration despite the fact that the situation is very common in sports.

Tanwar et al (2016) [5] investigated the effect of body acceleration on pulsatile blood flow through a catheterized artery. The blood was assumed to be a Newtonian fluid and the perturbation method was used to solve the problem. In their study, it was observed that the velocity of blood increases with the increase in body acceleration and the velocity decreases with the increase in phase angle.

Furthermore, a mathematical model for the blood flow through an overlapping stenosed artery under the effect of magnetic field was also studied by Parmar et al. (2013) [9]. The flow was assumed to be laminar, incompressible and fully developed. The blood was also assumed to follow Herschel-Bulkley fluids. The effects of magnetic fields and stenosis was discussed. Their findings found that magnetic fields and stenosis affect the normal flow of blood. 
MHD Arterial Blood Flow and Mass Transfer under the Presence of Stenosis, Body Acceleration and Chemical Reaction: A Case of Magnetic Therapy

Sharma and Gaur (2017) [14] reported that, blood is maintained in a delicate balance by a variety of chemical reactions, some that aid its coagulation and others its dissolution. Biologically blood reacts and is soluble at the arterial wall as arteries may be basically considered as a living tissues that need supply of metabolites including oxygen and removal of waste products. In this regard therefore, chemical reaction in blood flow exists, and it is therefore important know how are macromolecules transported or affected by the presence of chemical reaction. Modelling the combined effect of stenosis, body acceleration and chemical reactions for magnetic therapy (to the best of our knowledge) is missing in all literature despite of its manifestation in different situation like magnetic therapy in sports. The current study therefore aims at determining the combined effect of stenosis, body acceleration and chemical reaction in in magnetic therapies.

\section{Mathematical formulation}

We consider chemically reacting blood flowing through a stenosed artery in the presence of body acceleration and magnetic fields. The flow of blood is assumed to be two dimensional, unsteady, laminar, axisymmetric flow, fully developed and incompressible. We also assumethat the flow is under the influence of a constant electrical conductivity. Blood is assumed to be a Newtonian fluid. We further consider that $r=0$ is the axis of the axisymmetric flow where $\frac{\partial(.)}{\partial \theta}=0$ and $(u, w)$ are components of the velocityin $r, z$ directions respectively. Figure 1 shows the schematic diagram of the flow.

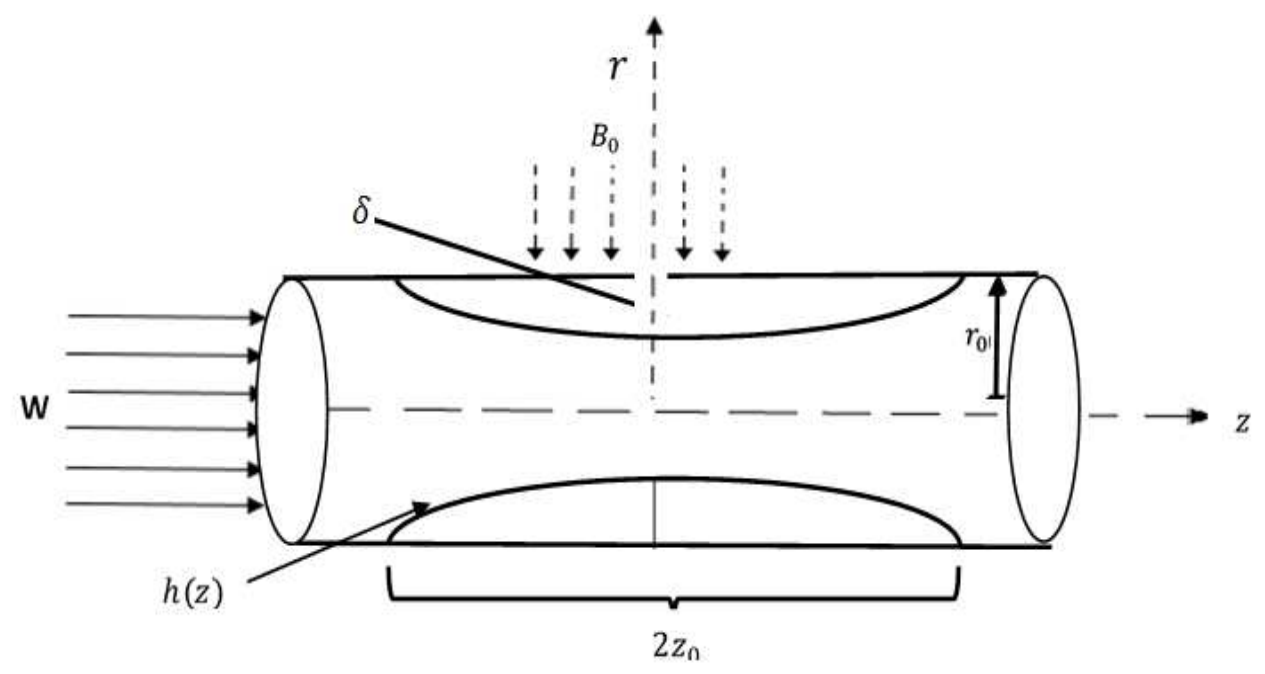

Figure 1: Schematic flow diagram

In cylindrical polar coordinate, under the mentioned assumptions, equations of continuity, motion and mass concentration reduce to:

$\frac{\partial u}{\partial r}+\frac{u}{r}+\frac{\partial w}{\partial z}=0$ 
Annord Mwapinga, Eunice Mureithi, James Makungu and Verdiana Masanja

$$
\begin{aligned}
& \rho\left(\frac{\partial u}{\partial t}+u \frac{\partial u}{\partial r}+w \frac{\partial u}{\partial z}\right)=-\frac{\partial P}{\partial r}+\mu\left(\frac{\partial^{2} u}{\partial r^{2}}+\frac{1}{r} \frac{\partial u}{\partial r}-\frac{u}{r^{2}}+\frac{\partial^{2} u}{\partial z^{2}}\right) \\
& \rho\left(\frac{\partial w}{\partial t}+u \frac{\partial w}{\partial r}+w \frac{\partial w}{\partial z}\right)=-\frac{\partial P}{\partial z}+\mu\left(\frac{\partial^{2} w}{\partial r^{2}}+\frac{1}{r} \frac{\partial w}{\partial r}+\frac{\partial^{2} w}{\partial z^{2}}\right)+G(t)-\sigma B_{0}^{2} w \\
& \rho\left(\frac{\partial C}{\partial t}+u \frac{\partial C}{\partial r}+w \frac{\partial C}{\partial z}\right)=D\left(\frac{\partial^{2} C}{\partial r^{2}}+\frac{1}{r} \frac{\partial C}{\partial r}+\frac{\partial^{2} C}{\partial z^{2}}\right)-\beta C
\end{aligned}
$$

where $r, z$ are the radial and axial directions whose corresponding velocities are respectively $u$ and $w . \rho$ is the density of the blood and $\mu$ is the blood's viscosity.P is the pressure, $\sigma$ is the electrical conductivity, $B_{0}$ is the applied magnetic field intensity, $G(t)$ is the body acceleration, $t$ is time, $C$ is the mass concentration, while $D$ and $\beta$ are the diffusion coeffient and chemical reaction parameters, respectively.

In the radial direction we assume that the pressure gradient is small due to the fact that the lumen radius of an artery is small in comparison to the pressure wave. Under such assumption therefore, the radial pressure gradient $\frac{\partial p}{\partial r} \approx 0$. Following Mustapha and Amin (2008), the pressure gradient for a human being in the axial direction can be written as

$$
-\frac{\partial P}{\partial z}=A_{0}+A_{1} \cos \left(n_{1} t\right)
$$

where $A_{0}$ is the steady state part of pressure gradient, $A_{1}$ is the amplitude of the pulsatile blood flow, that gives rise to systolic and diastolic pressure, $n_{1}=2 \pi f_{1}$, with $f_{1}$ being the pulse frequency. On other hand, according to Nagarani et al (2007), body acceleration may be given as

$$
G(t)=\rho a_{0} \cos \left(n_{2} t+\phi\right)
$$

where $\rho a_{0}$ is the amplitude of body acceleration, $n_{2}=2 \pi f_{2}$ with $f_{2}$ being body acceleration frequency, and $\phi$ is the phase angle.

The governing equations $1-4$ can now be written as follows;

$$
\begin{aligned}
\frac{\partial u}{\partial r}+\frac{u}{r}+\frac{\partial w}{\partial z}=0 & \\
\rho\left(\frac{\partial u}{\partial t}+u \frac{\partial u}{\partial r}+w \frac{\partial u}{\partial z}\right) & =+\mu\left(\frac{\partial^{2} u}{\partial r^{2}}+\frac{1}{r} \frac{\partial u}{\partial r}-\frac{u}{r^{2}}+\frac{\partial^{2} u}{\partial z^{2}}\right) \\
\rho\left(\frac{\partial w}{\partial t}+u \frac{\partial w}{\partial r}+w \frac{\partial w}{\partial z}\right) & =A_{0}+A_{1} \cos \left(n_{1} t\right)+\rho a_{0} \cos \left(n_{2} t+\phi\right)+\mu\left(\frac{\partial^{2} w}{\partial r^{2}}+\frac{1}{r} \frac{\partial w}{\partial r}+\frac{\partial^{2} w}{\partial z^{2}}\right) \\
& -\sigma B_{0}^{2} w \\
\rho\left(\frac{\partial C}{\partial t}+u \frac{\partial C}{\partial r}+w \frac{\partial C}{\partial z}\right) & =D\left(\frac{\partial^{2} C}{\partial r^{2}}+\frac{1}{r} \frac{\partial C}{\partial r}+\frac{\partial^{2} C}{\partial z^{2}}\right)-\beta C
\end{aligned}
$$


MHD Arterial Blood Flow and Mass Transfer under the Presence of Stenosis, Body Acceleration and Chemical Reaction: A Case of Magnetic Therapy

Following Das and Saha (2009), the geometry of stenosis (See Figure 1) can mathematically be expressed as follows;

$h(z)=\left\{\begin{array}{c}r_{0}-\delta\left(1+\cos \frac{\pi z}{2 z_{0}}\right), \quad-z_{0} \leq z \leq z_{0} \\ r_{0} \text { otherwise }\end{array}\right.$

where $h(z)$ represents the radius of the stenosed artery, $r_{0}$ is the radius of the normal artery, $2 z_{0}$ is the length of the stenosis and $\delta$ is the protuberance of the stenosis.

\section{Boundary and initial conditions}

It is assumed that there is no slip condition on the arterial wall, that is

$$
u(r, z, t)=0, \quad w(r, z, t)=0 \text { at } r=h(z)
$$

and at the center of the artery (at the line of symmetry) it is assumed that there is no shear rate and no radial flow, such that

$\frac{\partial w(r, z, t)}{\partial r}=0, \quad u(r, z, t)=0 \quad$ at $r=0$

For mass concentration, symmetric conditions are and concentration is considered uniform at the wall, Khan and Mohidul 2017.

$$
\frac{\partial C(r, z, t)}{\partial r}=0 \text { at } r=0 \quad \text { and } C(r, z, t)=0 \quad \text { at } \quad r=h(z)
$$

Since blood can flow even in the absence of magnetic field and body acceleration, it is therefore assumed that initially, there is non-zero velocity and concentration when $t=0$

$$
u(r, z, 0)=u_{0}, \quad w(r, z, 0)=w_{0}, C(r, z, 0)=C_{0}
$$

\section{Non- dimensionalisation of variables}

In this part we introduce the following non-dimensional variables. The variables $w_{c}$ and $r_{0}$ used are fluid characteristic velocity and distance. For this case, blood flowing in an artery, $w_{c}$ is the average blood velocity, and $r_{0}$ is the radius of the normal artery.

$$
\begin{gathered}
\eta=\frac{r}{r_{0}}, \quad z^{*}=\frac{z}{r_{0}}, \quad u^{*}=\frac{u}{w_{c}}, w^{*}=\frac{w}{w_{c}}, \quad \tau=\frac{t w_{c}}{r_{0}}, a_{0}^{*}=\frac{a_{0} r_{0}^{3}}{v^{2}}, A_{0}^{*}=\frac{A_{0} r_{0}^{3}}{\rho v^{2}} \\
A_{1}^{*}=\frac{A_{1} r_{0}^{3}}{\rho v^{2}}, \quad e=\frac{\delta}{r_{0}}, \quad C^{*}=\frac{C}{C_{0}}, \quad D^{*}=\frac{D}{v}, \quad \beta^{*}=\frac{\beta r_{0}^{2}}{v}, M=B_{0} r_{0} \sqrt{\frac{\sigma}{\mu}}
\end{gathered}
$$

Substituting these non-dimensional variables into equations 7-11 we get

Continuity equation

$$
\frac{\partial u^{*}}{\partial \eta}+\frac{u^{*}}{\eta}+\frac{\partial w^{*}}{\partial z^{*}}=0
$$

Equation of motion in the radial direction:

$$
\frac{\partial u^{*}}{\partial \tau}+u^{*} \frac{\partial u^{*}}{\partial \eta} w^{*} \frac{\partial u^{*}}{\partial z^{*}}=\frac{1}{R_{e}}\left(\frac{\partial^{2} u^{*}}{\partial \eta^{2}}+\frac{1}{\eta} \frac{\partial u^{*}}{\partial \eta}-\frac{u^{*}}{\eta^{2}}+\frac{\partial^{2} u^{*}}{\partial z^{* 2}}\right)
$$


Annord Mwapinga, Eunice Mureithi, James Makungu and Verdiana Masanja

Equation of motion in the axial direction

$$
\begin{aligned}
& \frac{\partial w^{*}}{\partial \tau}+u^{*} \frac{\partial w^{*}}{\partial \eta}+w^{*} \frac{\partial w^{*}}{\partial z^{*}}=A_{0}^{*}+A_{1}^{*} \cos \left(m_{1} \tau\right)+a_{0}^{*} \cos \left(m_{2} \tau+\phi\right) \\
& +\frac{1}{R_{e}}\left(\frac{\partial^{2} w^{*}}{\partial \eta^{2}}+\frac{1}{\eta} \frac{\partial w^{*}}{\partial \eta}+\frac{\partial^{2} w^{*}}{\partial z^{* 2}}\right)-\frac{M^{2}}{R_{e}} w^{*}
\end{aligned}
$$

Mass concentration equation

$$
\frac{\partial C^{*}}{\partial \tau}+u^{*} \frac{\partial C^{*}}{\partial \eta}+w^{*} \frac{\partial C^{*}}{\partial z^{*}}=D^{*}\left(\frac{\partial^{2} C^{*}}{\partial \eta^{2}}+\frac{1}{\eta} \frac{\partial C^{*}}{\partial \eta}+\frac{\partial^{2} C^{*}}{\partial z^{* 2}}\right)-\beta C^{*}
$$

where $R_{e}=\frac{w_{c} r_{0}}{v}, D^{*}=\frac{D}{v}=\frac{1}{S c} S c$ are, respectively, the Reynolds number, and Schmidt number, and $M=B_{0} r_{0} \sqrt{\frac{\sigma}{\mu}}$ is the Hartmann number. The mass concentration equation becomes

$$
\frac{\partial C^{*}}{\partial \tau}+u^{*} \frac{\partial C^{*}}{\partial \eta}+w^{*} \frac{\partial C^{*}}{\partial z^{*}}=\frac{1}{S c}\left(\frac{\partial^{2} C^{*}}{\partial \eta^{2}}+\frac{1}{\eta} \frac{\partial C^{*}}{\partial \eta}+\frac{\partial^{2} C^{*}}{\partial z^{* 2}}\right)-\beta C^{*}
$$

The geometry of stenosis (Equation 5) in dimensionless form becomes

$$
H\left(z^{*}\right)=\left\{\begin{array}{c}
1-e\left(1+\cos \left(\frac{\pi z^{*}}{2}\right)\right) \text { for }-1 \leq z^{*} \leq 1 \\
\text { otherwise }
\end{array}\right.
$$

The boundary and initial conditions $12-15$ in dimensionless form become:

$$
\begin{gathered}
\left\{\begin{array}{l}
u^{*}\left(\eta, z^{*}, \tau\right)=0, \quad w^{*}\left(\eta, z^{*}, \tau\right)=0 \quad \text { at } \eta=H\left(z^{*}\right) \\
\frac{\partial w\left(\eta, z^{*}, \tau\right)}{\partial \eta}=0, \quad u^{*}\left(\eta, z^{*}, \tau\right)=0 \quad \text { at } \eta=0
\end{array}\right. \\
C^{*}\left(\eta, z^{*}, \tau\right)=0, \quad \text { at } \eta=H\left(z^{*}\right), \quad \frac{\partial C^{*}\left(\eta, z^{*}, \tau\right)}{\partial \eta}=0, \quad \text { at } \eta=0 \\
u^{*}\left(\eta, z^{*}, 0\right)=u_{0}^{*}, \quad w^{*}\left(\eta, z^{*}, 0\right)=w_{0}^{*}, \quad C^{*}\left(\eta, z^{*}, 0\right)=1,
\end{gathered}
$$

In this work, the initial condition for velocity was obtained from the steady state of the equation of motion as

$$
w(\eta)=\left(\frac{A_{0}+A_{1}}{4}\right)\left(1-\eta^{2}\right)=w_{0}
$$

\section{Solutions}

\subsection{Radial coordinate transformation}

In this section we are going to transform the equations from cylindrical to rectangular domain. Assuming that the artery is cylindrical with stenosis, we transform the constriction by introducing another variable $\xi$ such that 
MHD Arterial Blood Flow and Mass Transfer under the Presence of Stenosis, Body Acceleration and Chemical Reaction: A Case of Magnetic Therapy

$$
\xi=\frac{\eta}{H(z)}
$$

This suitable radial coordinate transformation helps to map the constricted domain intoa rectangular one. That is, this has the effect of immobilizing the arterial wall in thetransformed coordinate $\xi$. Using the above transformation, re-arranging and dropping the asterisks, theequations of continuity, motion, and mass concentration transfer become:

$$
\begin{aligned}
& \frac{\partial u}{H \partial \xi}+\frac{u}{H \xi}+\frac{\partial w}{\partial z}-\frac{\xi}{H} \frac{d H}{d z} \frac{\partial w}{\partial \xi}=0 \\
& \frac{\partial u}{\partial \tau}=-\frac{u}{H} \frac{\partial u}{\partial \xi}-w\left[\frac{\partial u}{\partial z}-\frac{\xi}{H} \frac{d H}{d z} \frac{\partial u}{\partial \xi}\right]+\frac{1}{R_{e}} \frac{1}{H^{2}}\left(\frac{\partial^{2} u}{\partial \xi^{2}}+\frac{1}{\xi} \frac{\partial u}{\partial \xi}-\frac{u}{\xi^{2}}\right)+\frac{1}{R_{e}}\left[\frac{\partial^{2} u}{\partial z^{2}}-\frac{2 \xi}{H} \frac{d H}{d z} \frac{\partial^{2} u}{\partial \xi \partial z}\right. \\
& \left.-\frac{\xi}{H} \frac{d^{2} H}{d z^{2}} \frac{\partial u}{\partial \xi}+\frac{\xi^{2}}{H^{2}}\left(\frac{d H}{d z}\right)^{2} \frac{\partial^{2} u}{\partial \xi^{2}}+\frac{3 \xi}{H^{2}}\left(\frac{d H}{d z}\right)^{2} \frac{\partial u}{\partial \xi}\right] \\
& \frac{\partial w}{\partial \tau}=-\frac{u}{H} \frac{\partial w}{\partial \xi}-w\left[\frac{\partial w}{\partial z}-\frac{\xi}{H} \frac{d H}{d z} \frac{\partial w}{\partial \xi}\right]+A_{0}+A_{1} \cos \left(m_{1} \tau\right)+\cos \left(m_{2} \tau+\phi\right) \\
& +\frac{1}{R_{e}} \frac{1}{H^{2}}\left(\frac{\partial^{2} w}{\partial \xi^{2}}+\frac{1}{\xi} \frac{\partial w}{\partial \xi}\right)+\frac{1}{R_{e}}\left[\frac{\partial^{2} w}{\partial z^{2}}-\frac{2 \xi}{H} \frac{d H}{d z} \frac{\partial^{2} w}{\partial \xi \partial z}-\frac{\xi}{H} \frac{d^{2} H}{d z^{2}} \frac{\partial w}{\partial \xi}+\frac{\xi^{2}}{H^{2}}\left(\frac{d H}{d z}\right)^{2} \frac{\partial^{2} w}{\partial \xi^{2}}\right. \\
& \left.+\frac{3 \xi}{H^{2}}\left(\frac{d H}{d z}\right)^{2} \frac{\partial w}{\partial \xi}\right]-\frac{1}{R_{e}} M^{2} w \\
& \frac{\partial C}{\partial \tau}=-\frac{u}{H} \frac{\partial C}{\partial \xi}-w\left[\frac{\partial C}{\partial z}-\frac{\xi}{H} \frac{d H}{d z} \frac{\partial C}{\partial \xi}\right]+\frac{1}{S C}\left(\frac{\partial^{2} C}{\partial \xi^{2}}+\frac{1}{\xi} \frac{\partial C}{\partial \xi}\right)+\frac{1}{S C}\left[\frac{\partial^{2} C}{\partial z^{2}}-\frac{2 \xi}{H} \frac{d H}{d z} \frac{\partial^{2} C}{\partial \xi \partial z}-\frac{\xi}{H} \frac{d^{2} H}{d z^{2}} \frac{\partial C}{\partial \xi}\right. \\
& \left.+\frac{\xi^{2}}{R^{2}}\left(\frac{d H}{d z}\right)^{2} \frac{\partial^{2} C}{\partial \xi^{2}}+\frac{3 \xi}{H^{2}}\left(\frac{d H}{d z}\right)^{2} \frac{\partial C}{\partial \xi}\right]-\beta C
\end{aligned}
$$

Similarly, the boundary conditions 22-24 under the given radial coordinate transformation, become:

$$
\begin{aligned}
& \left\{\begin{array}{l}
u(\xi, z, \tau)=0, \quad w(\xi, z, \tau)=0 \quad \text { at } \xi=1 \\
\frac{\partial w(\xi, z, \tau)}{\partial \xi}=0, u(\xi, z, \tau)=0 \quad \text { at } \xi=0
\end{array}\right. \\
& C(\xi, z, \tau)=0, \quad \text { at } \xi=1, \quad \frac{\partial C(\xi, z, \tau)}{\partial \xi}=0, \quad \text { at } \xi=0 \\
& u(\xi, z, 0)=u_{0}, \quad w(\xi, z, 0)=w_{0}, C(\xi, z, 0)=c_{0},
\end{aligned}
$$

Initial velocity $w_{0}$ becomes

$$
w_{0}=\left(\frac{A_{0}+A_{1}}{4}\right)\left(1-(H \xi)^{2}\right)
$$

\subsection{Radial velocity}

To obtain the radial momentum, we use the continuity equation, we therefore multiply equation 27 by $\xi H$ and integratethe resulting equation with respect to $\xi$. This gives 
Annord Mwapinga, Eunice Mureithi, James Makungu and Verdiana Masanja

$u(\xi, z, \tau)=\xi \frac{d H}{d z} w-\frac{2}{H} \frac{d H}{d z} \int_{0}^{\xi} w \xi d \xi-\frac{H}{\xi} \int_{0}^{\xi} \xi \frac{\partial w}{\partial z} d \xi$

Now, applying the boundary condition 31 , we obtain

$\int_{0}^{1} \frac{2}{H} \frac{d H}{d z} w \xi d \xi=-\int_{0}^{1} \xi \frac{\partial w}{\partial z} d \xi$

Comparing integrals and the integrands of equation 36 , we have;

$\frac{\partial w}{\partial z}=-\frac{2}{H} \frac{d H}{d z} w$

Substituting equation 37 into 35 we obtain

$u(\xi, z, \tau)=\xi \frac{d H}{d z} w$

Equation 38 is the radial velocity component which has to be calculated. However, equation 38 will now be substituted into equations 29 and 30 and obtain:

$$
\begin{aligned}
& \frac{\partial w}{\partial \tau}=\left(-\frac{\xi}{H} \frac{d H}{d z} w\right) \frac{\partial w}{\partial \xi}-w\left[\frac{\partial w}{\partial z}-\frac{\xi}{H} \frac{d H}{d z} \frac{\partial w}{\partial \xi}\right]+A_{0}+A_{1} \cos \left(m_{1} \tau\right)+\cos \left(m_{2} \tau+\phi\right) \\
& +\frac{1}{R_{e}} \frac{1}{H^{2}}\left(\frac{\partial^{2} w}{\partial \xi^{2}}+\frac{1}{\xi} \frac{\partial w}{\partial \xi}\right)+\frac{1}{R_{e}}\left[\frac{\partial^{2} w}{\partial z^{2}}-\frac{2 \xi}{H} \frac{d H}{d z} \frac{\partial^{2} w}{\partial \xi \partial z}-\frac{\xi}{H} \frac{d^{2} H}{d z^{2}} \frac{\partial w}{\partial \xi}+\frac{\xi^{2}}{H^{2}}\left(\frac{d H}{d z}\right)^{2} \frac{\partial^{2} w}{\partial \xi^{2}}+\frac{3 \xi}{H^{2}}\left(\frac{d H}{d z}\right)^{2} \frac{\partial w}{\partial \xi}\right] \\
& \quad-\frac{1}{R_{e}} M^{2} w \\
& \frac{\partial C}{\partial \tau}=\left(-\frac{\xi}{H} \frac{d H}{d z} w\right) \frac{\partial C}{\partial \xi}-w\left[\frac{\partial C}{\partial z}-\frac{\xi}{H} \frac{d H}{d z} \frac{\partial C}{\partial \xi}\right]+\frac{1}{S c}\left(\frac{\partial^{2} C}{\partial \xi^{2}}+\frac{1}{\xi} \frac{\partial C}{\partial \xi}\right)+\frac{1}{S C}\left[\frac{\partial^{2} C}{\partial z^{2}}-\frac{2 \xi}{H} \frac{d H}{d z} \frac{\partial^{2} C}{\partial \xi \partial z}-\frac{\xi}{H} \frac{d^{2} H}{d z^{2}} \frac{\partial C}{\partial \xi}\right. \\
& \left.+\frac{\xi^{2}}{H^{2}}\left(\frac{d H}{d z}\right)^{2} \frac{\partial^{2} C}{\partial \xi^{2}}+\frac{3 \xi}{H^{2}}\left(\frac{d H}{d z}\right)^{2} \frac{\partial C}{\partial \xi}\right]-\beta C
\end{aligned}
$$

\subsection{Finite difference schemes}

Equations (39) and (40) are solved numerically using the FDM.The finite difference discretization is based on central differences for space and forward difference for time.

$$
\left.\begin{array}{rl}
\frac{\partial w}{\partial \xi} & =\frac{w_{i, j+1}^{k}-w_{i, j-1}^{k}}{2(\Delta \xi)}, \quad \frac{\partial w}{\partial z}=\frac{w_{i+1, j}^{k}-w_{i-1, j}^{k}}{2(\Delta z)}, \frac{\partial C}{\partial z}=\frac{C_{i+1, j}^{k}-C_{i-1, j}^{k}}{2(\Delta z)} \frac{\partial C}{\partial \xi}=\frac{C_{i, j+1}^{k}-C_{i, j-1}^{k}}{2(\Delta \xi)} \\
\frac{\partial^{2} w}{\partial \xi^{2}} & =\frac{w_{i, j+1}^{k}-2 w_{i, j}^{k}+w_{i, j-1}^{k}}{(\Delta \xi)^{2}}, \frac{\partial^{2} w}{\partial z^{2}}=\frac{w_{i+1, j}^{k}-2 w_{i, j}^{k}+w_{i-1, j}^{k}}{(\Delta z)^{2}}, \frac{\partial^{2} C}{\partial z^{2}}=\frac{C_{i+1, j}^{k}-2 C_{i, j}^{k}+C_{i-1, j}^{k}}{(\Delta z)^{2}} \\
\frac{\partial^{2} C}{\partial \xi^{2}} & =\frac{C_{i, j+1}^{k}-2 C_{i, j}^{k}+C_{i, j-1}^{k}}{(\Delta \xi)^{2}}, \text { and for time we have } \frac{\partial w}{\partial \tau}=\frac{w_{i, j}^{k+1}-w_{i, j}^{k}}{\Delta \tau}, \frac{\partial C}{\partial \tau}=\frac{C_{i, j}^{k+1}-C_{i, j}^{k}}{\Delta \tau}
\end{array}\right\}
$$


MHD Arterial Blood Flow and Mass Transfer under the Presence of Stenosis, Body Acceleration and Chemical Reaction: A Case of Magnetic Therapy

We now substitute the finite difference approximationsfrom equation 42 to equations 39 and 40 and we make $w_{i, j}^{k+1}$ and $C_{i, j}^{k+1}$ the subject, and obtain:

$$
\begin{aligned}
& w_{i, j}^{k+1}=w_{i, j}^{k}+\Delta \tau\left\{\left(-\frac{\xi_{j}}{H_{i}}\left(\frac{d H}{d z}\right)_{i} w_{i, j}^{k}\right)\left(\frac{w_{i, j+1}^{k}-w_{i, j-1}^{k}}{2(\Delta \xi)}\right)-w_{i, j}^{k}\left[\frac{w_{i+1, j}^{k}-w_{i-1, j}^{k}}{2(\Delta z)}-\left(\frac{\xi_{j}}{H_{i}}\left(\frac{d H}{d z}\right)_{i}\right) \frac{w_{i, j+1}^{k}-w_{i, j-1}^{k}}{2(\Delta \xi)}\right]\right. \\
& +\frac{1}{R_{e}}\left(\frac{w_{i+1, j}^{k}-2 w_{i, j}^{k}+w_{i-1, j}^{k}}{(\Delta z)^{2}}\right)-\frac{1}{R_{e}} \frac{2 \xi_{j}}{H_{i}}\left(\frac{d H}{d z}\right)_{i}\left(\frac{w_{i+1, j+1}^{k}+w_{i-1, j-1}^{k}-w_{i-1, j+1}^{k}-w_{i+1, j-1}^{k}}{4(\Delta \xi)(\Delta z)}\right) \\
& -\frac{1}{R_{e}} \frac{\xi_{j}}{H_{i}}\left(\frac{d^{2} H}{d z^{2}}\right)_{i} \frac{w_{i, j+1}^{k}-w_{i, j-1}^{k}}{2(\Delta \xi)}+\frac{1}{R_{e}} \frac{\xi_{j}^{2}}{H_{i}^{2}}\left(\frac{d H}{d z}\right)_{i}^{2}\left(\frac{w_{i, j+1}^{k}-2 w_{i, j}^{k}+w_{i, j-1}^{k}}{(\Delta \xi)^{2}}\right) \\
& \left.+\frac{1}{R_{e}} \frac{3 \xi_{j}}{H_{i}^{2}}\left(\frac{d H}{d z}\right)_{i}^{2}\left(\frac{w_{i, j+1}^{k}-w_{i, j-1}^{k}}{2(\Delta \xi)}\right)-\frac{1}{R_{e}} M^{2} w_{i, j}^{k}\right\} \\
& C_{i, j}^{k+1}=C_{i, j}^{k}+\Delta \tau\left\{\left(\xi_{j}\left(\frac{d H}{d z}\right)_{i} w_{i, j}^{k}\right) \frac{C_{i, j+1}^{k}-C_{i, j-1}^{k}}{2(\Delta \xi)}-w_{i, j}^{k}\left[\frac{C_{i, j+1}^{k}-C_{i, j-1}^{k}}{2(\Delta z)}-\left(\frac{\xi_{j}}{H_{i}}\left(\frac{d H}{d z}\right)_{i}\right) \frac{C_{i, j+1}^{k}-C_{i, j-1}^{k}}{2(\Delta \xi)}\right]\right. \\
& +\frac{1}{S c}\left[\frac{C_{i, j+1}^{k}-2 C_{i, j}^{k}+C_{i, j-1}^{k}}{(\Delta \xi)^{2}}+\frac{1}{\xi_{i}}\left(\frac{C_{i, j+1}^{k}-C_{i, j-1}^{k}}{2(\Delta \xi)}\right)\right]+\frac{1}{S c}\left[\frac{C_{i+1, j}^{k}-2 C_{i, j}^{k}+C_{i-1, j}^{k}}{(\Delta z)^{2}}\right. \\
& -\frac{2 \xi_{j}}{H_{i}}\left(\frac{d H}{d z}\right)_{i}\left(\frac{C_{i+1, j+1}^{k}+C_{i-1, j-1}^{k}-C_{i-1, j+1}^{k}-C_{i+1, j-1}^{k}}{4(\Delta \xi)(\Delta z)}\right)-\frac{\xi_{j}}{H_{i}}\left(\frac{d^{2} H}{d z^{2}}\right)_{i}\left(\frac{C_{i, j+1}^{k}-C_{i, j-1}^{k}}{2(\Delta \xi)}\right) \\
& \left.+\frac{\xi_{j}}{H_{i}}\left(\frac{d^{2} H}{d z^{2}}\right)_{i} \frac{C_{i, j+1}^{k}-C_{i, j-1}^{k}}{2(\Delta \xi)}\right\}-\Delta \tau \beta C_{i, j}^{k}+\frac{\xi_{j}^{2}}{H_{i}^{2}}\left(\frac{d H}{d z}\right)_{i}^{2}\left(\frac{C_{i, j+1}^{k}-2 C_{i, j}^{k}+C_{i, j-1}^{k}}{(\Delta \xi)^{2}}\right) \\
& \left.\left.+\frac{3 \xi_{j}}{H_{i}^{2}}\left(\frac{d H}{d z}\right)^{2}\left(\frac{C_{i, j+1}^{k}-w_{i, j-1}^{k}}{2(\Delta \xi)}\right)\right]-\beta C_{i, j}^{k}\right\}
\end{aligned}
$$

where we define

$z_{i}=(i-1) \Delta z, \quad i=1,2, \ldots, M+1 ; \xi_{j}=(j-1) \Delta \xi, \quad j=1,2, \ldots, N+1$

$\tau_{k}=(i-1) \Delta \tau, \quad k=1,2, \ldots \quad$ Here $\Delta z$ and $\Delta \xi$ represent the spatial increments, respectively, in the axial and radial directions while $\Delta \tau$ represents a small increment in time. We also discretize the boundary conditions as follows:

The Neumann boundary condition at $\xi=0$ we have

$$
\frac{\partial w}{\partial \xi}=\frac{w_{i, j+1}^{k}-w_{i, j-1}^{k}}{2(\Delta \xi)}=0
$$

This gives

$w_{i, j+1}^{k}-w_{i, j-1}^{k}=0$ this implies that $w_{i, j+1}^{k}=w_{i, j-1}^{k}$ now at $\xi=0$ means that $j=1$, thus this means $w_{i, 2}^{k}=w_{i, 0}^{k}$. However, $w_{i, 0}^{k}$ is outside the domain of interest. It is therefore the ghost point. To get rid of this ghost point we further approximate the derivative for the interval $\Delta \xi$ as follows 
Annord Mwapinga, Eunice Mureithi, James Makungu and Verdiana Masanja

$$
\left.\frac{\partial w}{\partial \xi}\right|_{j=1} \cong \frac{w_{i, j+1}^{k}-w_{i, j}^{k}}{\Delta \xi}=0
$$

This gives $w_{i, 2}^{k}=w_{i, 1}^{k}$, we therefore have

$u_{i, 1}^{k}=u_{i, N+1}^{k}=w_{i, N+1}^{k}=0, \quad w_{i, j}^{1}=w_{0}, C_{i, j}^{1}=c_{0}, w_{i, 2}^{k}=w_{i, 1}^{k}, C_{i, 2}^{k}=C_{i, 1}^{k}$

The axial initial velocity is therefore discretized as $w_{0}=\left(\frac{A_{0}+A_{1}}{4}\right)\left(1-\left(H \xi_{i}\right)^{2}\right)$

\section{Results and discussion}

\subsection{Numerical simulation}

In this section we implemented the numerical model (equations 42, 43, and 46) and performed computer simulations using MATLAB codes. To maintain stability, we ensured that $0<\frac{\Delta \tau}{(\Delta \xi)^{2}} \leq 0.5$. Forconvenience, constants were wisely chosen as follows; $\mathrm{A}_{0}=1, \mathrm{~A}_{1}=0.5, \mathrm{~m}_{1}=1 \mathrm{~m}_{2}=1, \phi=0.6, \mathrm{e}=0.1, \mathrm{~B}_{0}=1, \mathrm{a}_{0}=1, \mathrm{~S}_{\mathrm{c}}=3, \operatorname{Re}=3, \mathrm{z}=$ 0.2

$\tau=0.1990, \Delta \tau=0.001$. Some of these parameters were varied to observe their effects as illustrated in Fig. 1 to Fig. 23.

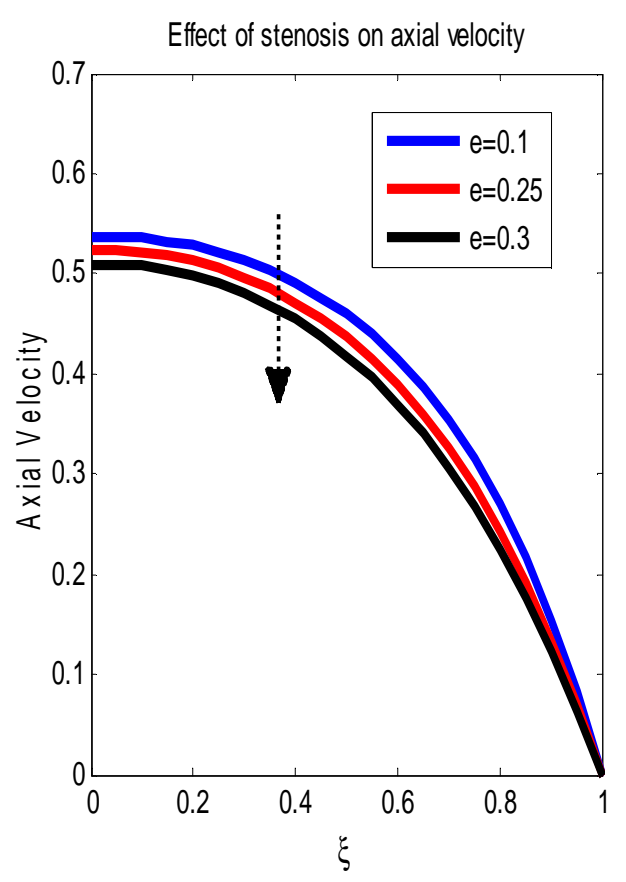

Figure 2:

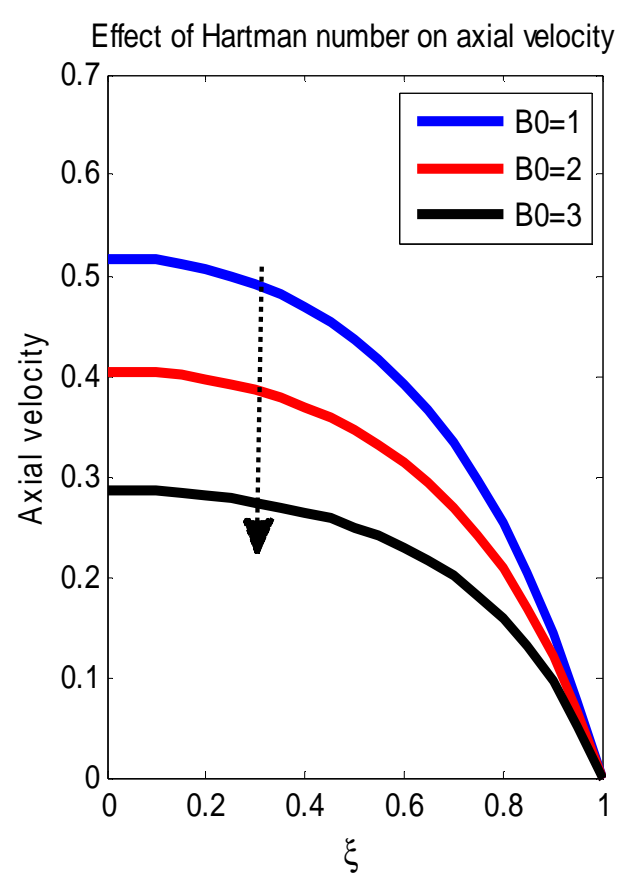

Figure 3: 
MHD Arterial Blood Flow and Mass Transfer under the Presence of Stenosis, Body Acceleration and Chemical Reaction: A Case of Magnetic Therapy

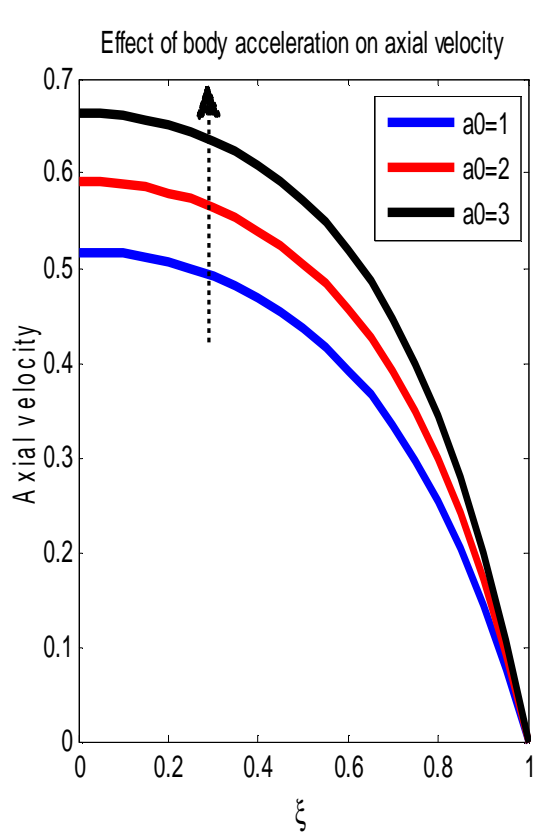

Figure 4:

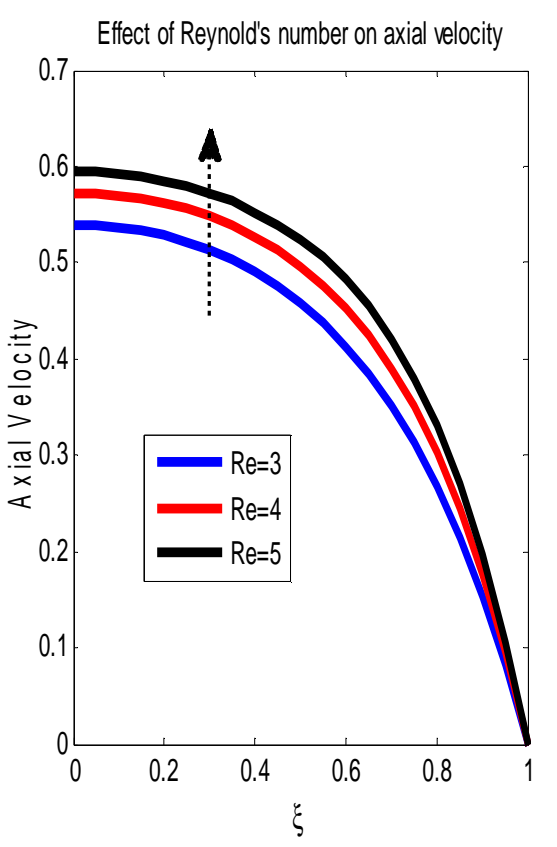

Figure 5:

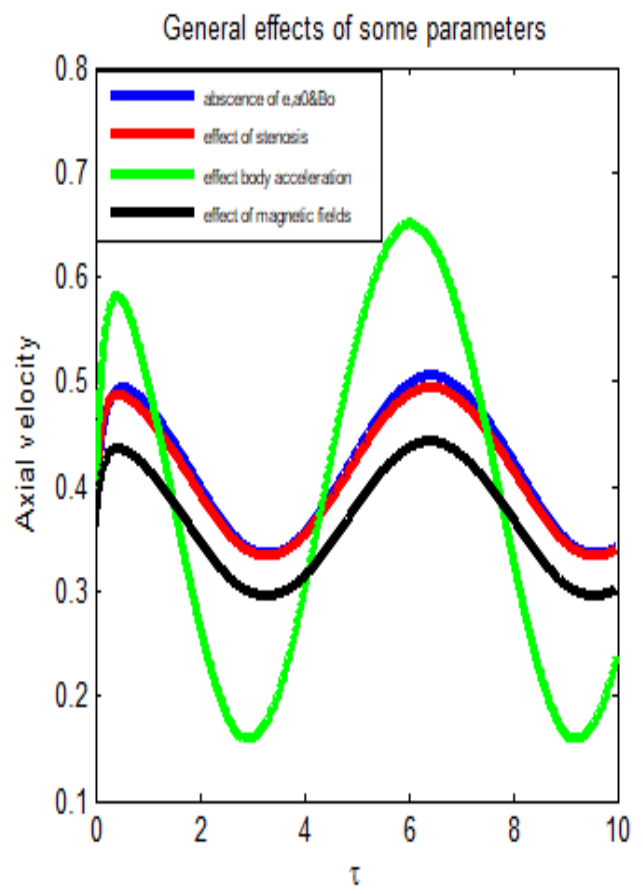

Figure 6:

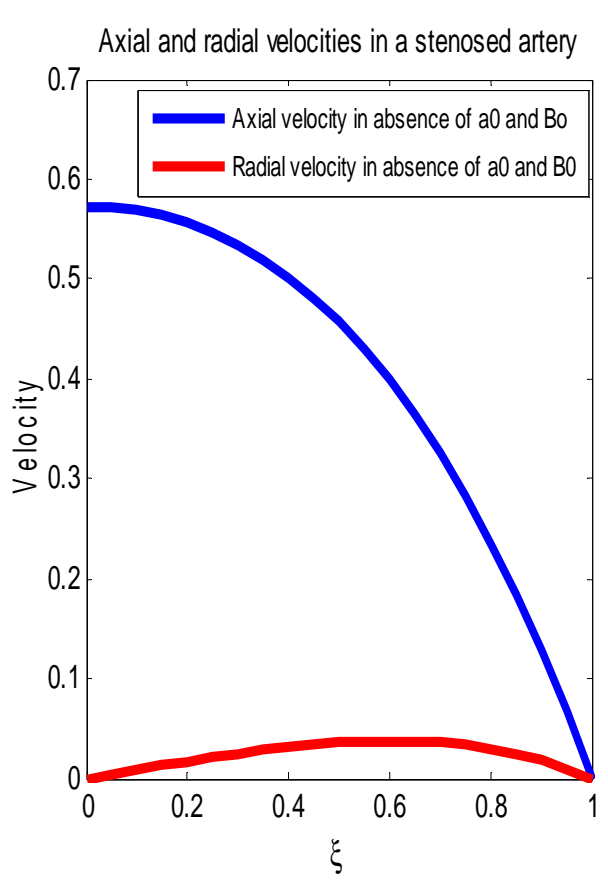

Figure 7: 
Annord Mwapinga, Eunice Mureithi, James Makungu and Verdiana Masanja

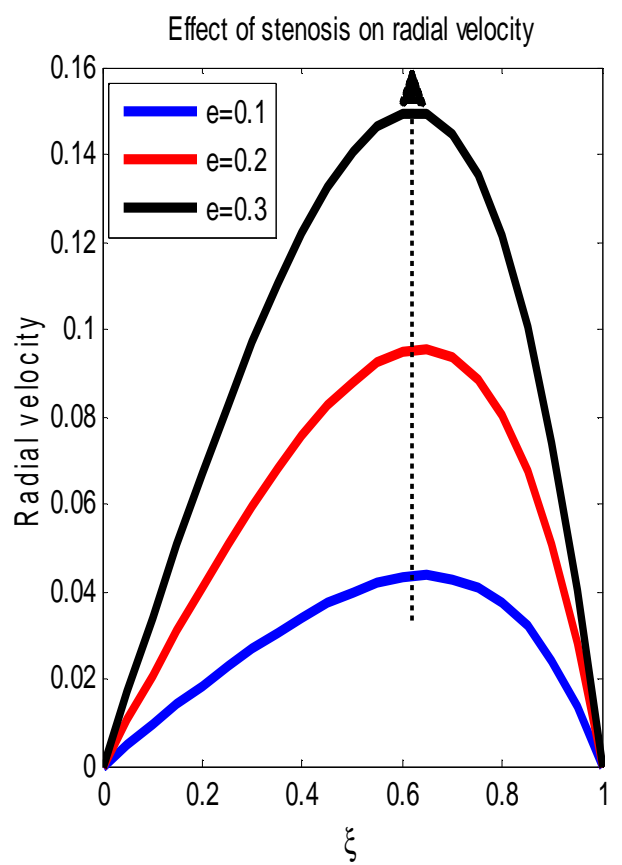

Figure 8:

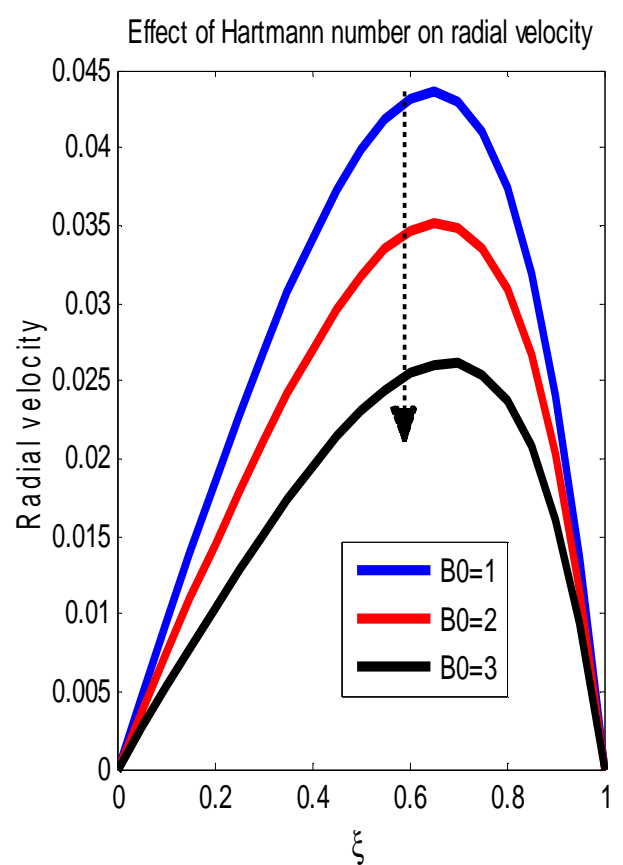

Figure 9:

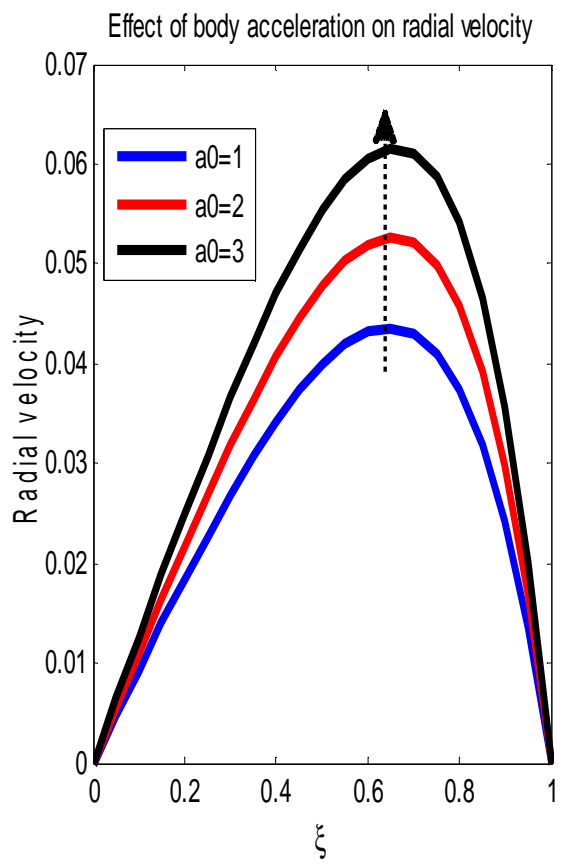

Figure 10:

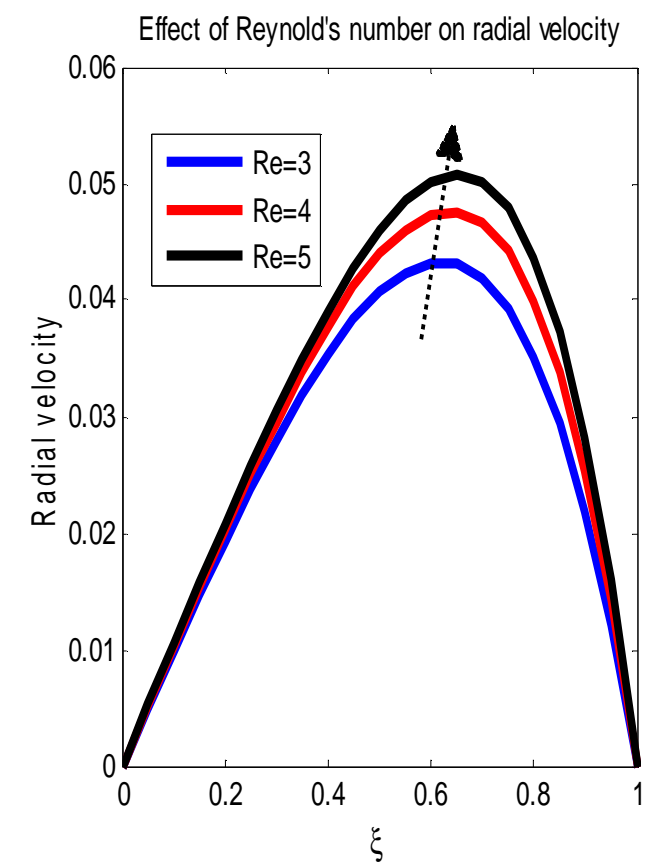

Figure 11: 
MHD Arterial Blood Flow and Mass Transfer under the Presence of Stenosis, Body Acceleration and Chemical Reaction: A Case of Magnetic Therapy

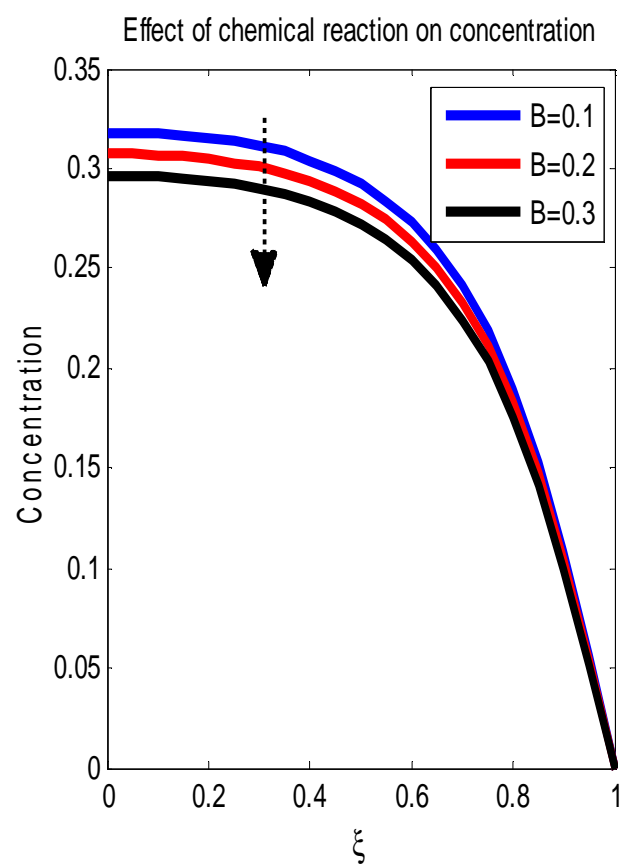

Figure 12:

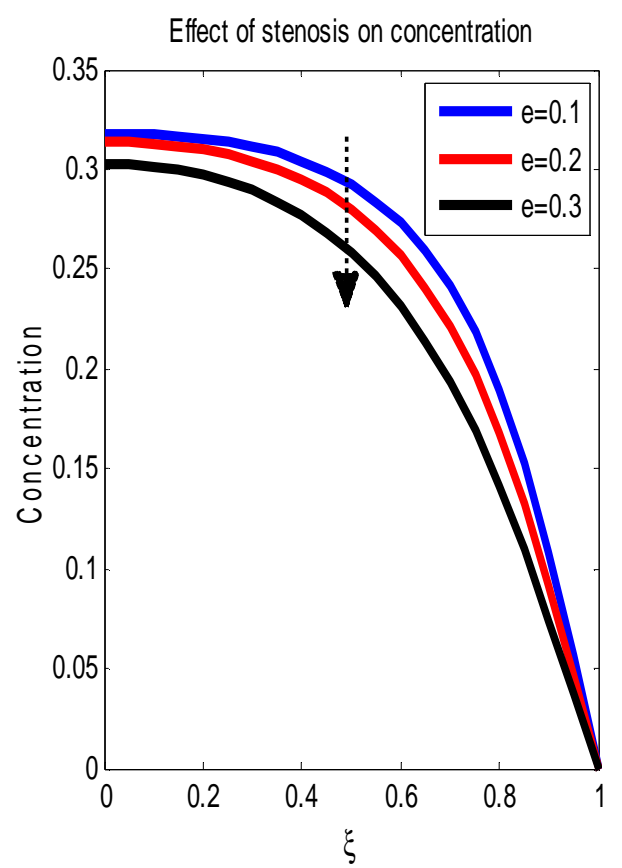

Figure 14:

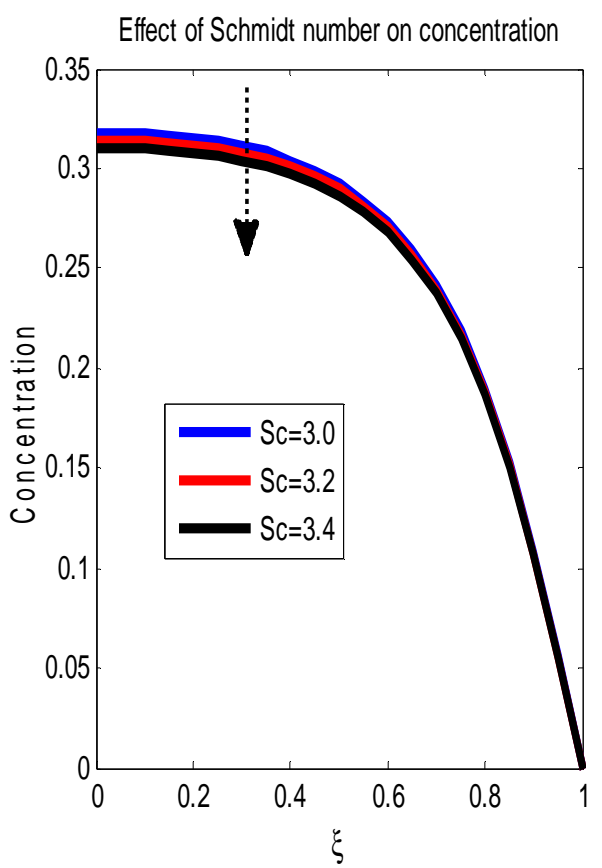

Figure 13:

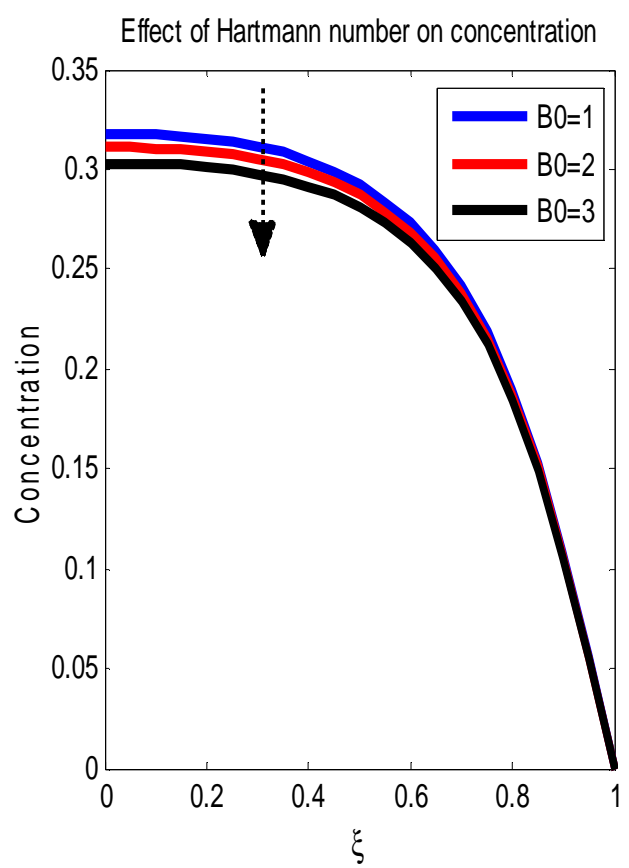

Figure 15: 
Annord Mwapinga, Eunice Mureithi, James Makungu and Verdiana Masanja

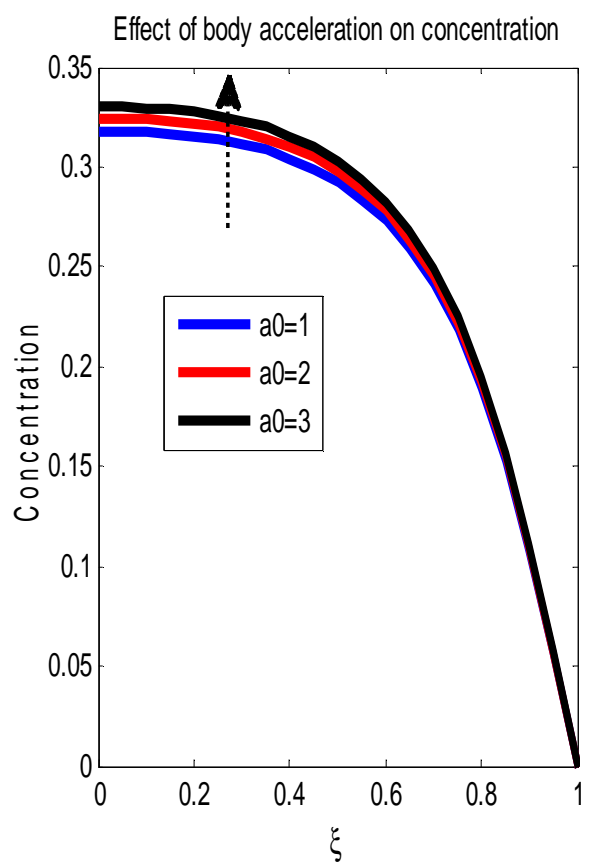

Figure 16:

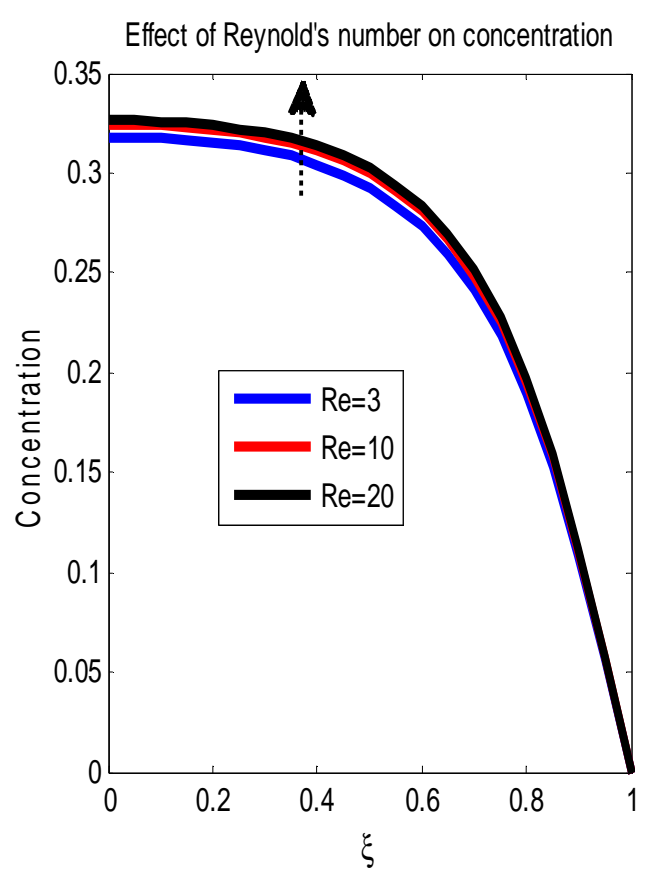

Figure 17:

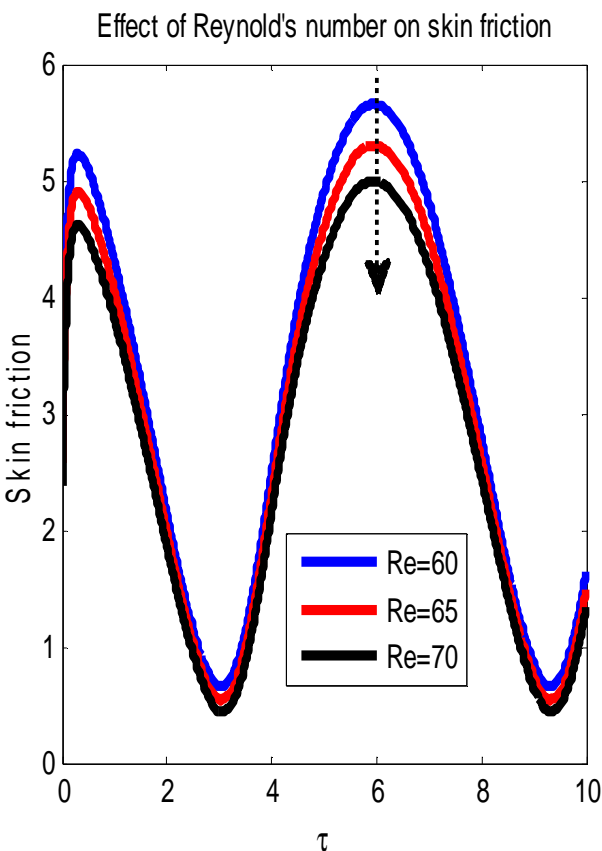

Figure 19: 
MHD Arterial Blood Flow and Mass Transfer under the Presence of Stenosis, Body Acceleration and Chemical Reaction: A Case of Magnetic Therapy

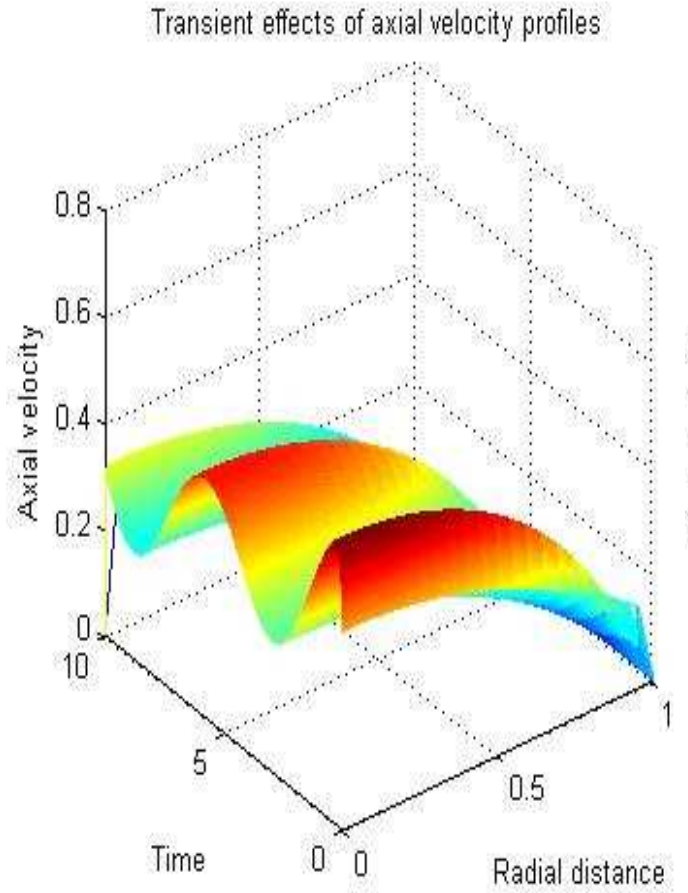

Figure 20:

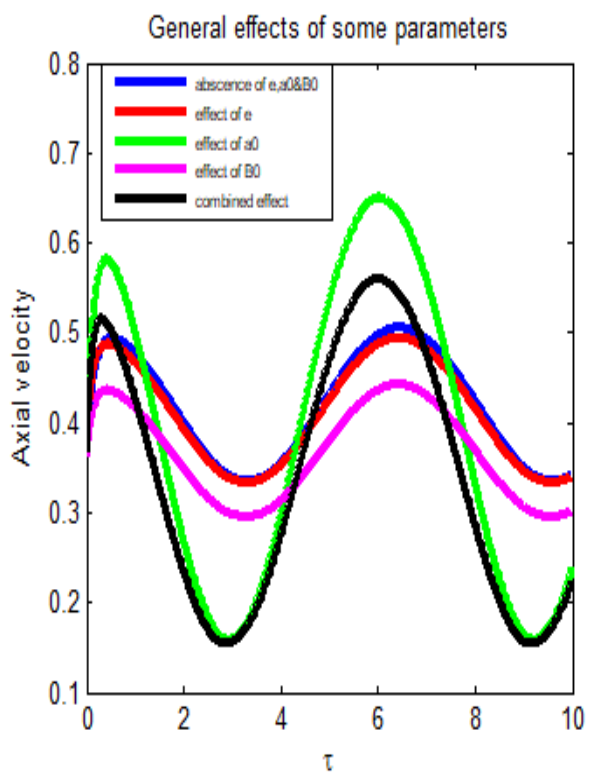

Figure 22:

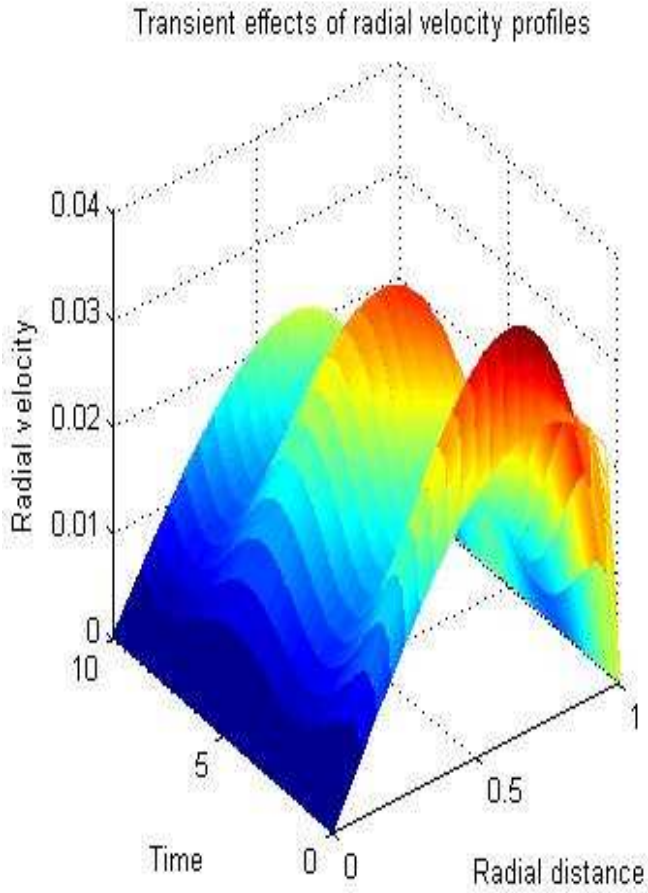

Figure 21:

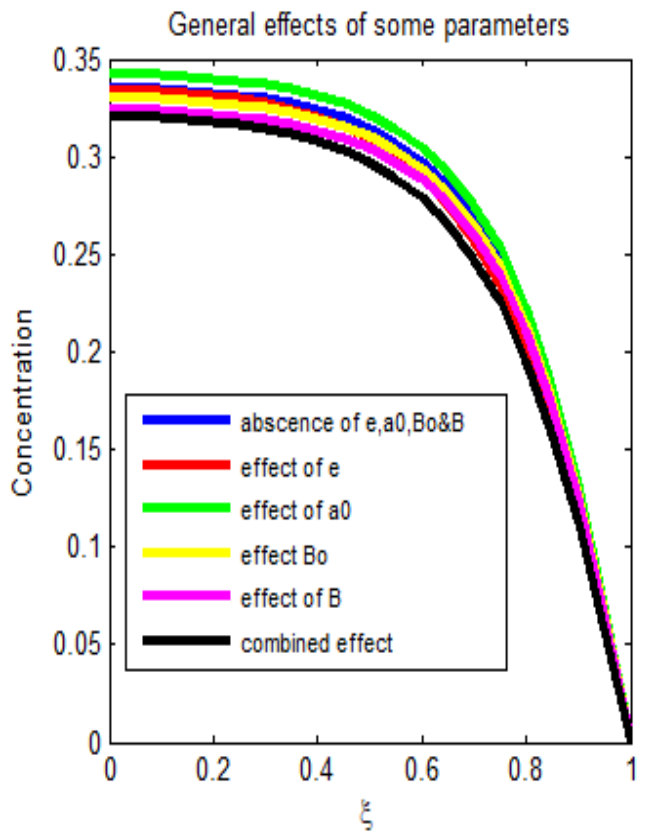

Figure 23: 
Annord Mwapinga, Eunice Mureithi, James Makungu and Verdiana Masanja

\subsection{Discussion of the simulations}

The effect of Hartmann number on axial velocity profiles is illustrated by figure 3. From the graph we observe that as the Hartmann number increases, the velocity decreases due to the fact that as the magnetic fields is applied to the body, the Lorentz force tend to oppose the flow of blood. The Lorentz force is able to oppose the motion of the blood flow because blood consists of red blood cells which contains ions. Similar results were obtained by Kumari et al, (2019) [1] and Sharma et al (2019) [4] who considered the navier slip.

Figure 4 shows the effect of increasing body acceleration on axial velocity. From the graph it is observed that for the fixed values of Hartmann number and height of stenosis, axial velocity increases as body acceleration increases. The increase in velocity is due to the reason that body acceleration increases the heart beats and the pulse rate. When the body is subjected to body acceleration, the heart speeds up to pumping blood so that more blood can reach the muscles.

This study also investigated the effect of Reynolds number on axial velocity. From the graph we see that, as Reynolds numbers increase, the axial velocity increases. The increase in Reynold's number implies more increase of inertial forces than the viscous forces. Thus, as the inertial force increases, the velocity increases.

Figure 7 shows both, the axial velocity and the radial velocity. From the graph we see that the axial velocity is higher than the radial velocity. This is due to the fact that the pressure gradient is more dominant in the axial direction than in radial direction. On the other hand, we also observe that, the axial velocity is maximum along the axis of symmetry and it is zero on the boundary. The axial velocity is decreasing as we move towards the boundary because of the no slip condition at the boundary. The radial velocity is zero along the line of symmetry because there is no radial flow along that axis. Also, it is zero at the boundary, to satisfy the no slip condition. It is very interesting to note that it has been revealed that increase in the severity of stenosis, decreases the axial velocity but increase the radial velocity. The radial velocity increases as compensation to the axial velocity which decreases. However, this may medically endanger a person by harming the arterial wall for prolonged situation.

The effect of Hartman number, body acceleration and Reynolds number on radial velocity are shown in figures 9,10 and 11 respectively. The effect of increasing these parameters on the radial velocity is observed to be the same as it is manifested on the axial velocity.

The effect of increasing the chemical reaction parameter on concentration profile is illustrated in figure 12. From the graph, we clearly observe that, as the chemical reaction parameter increases, the concentration decreases. The decrease of the concentration is due to the fact that the presence of chemical reaction acts as the consumption or destructive agent of chemical species. This leads to the reduction of the concentration.

Figure 13 shows the effect of increasing the Schmidt number on concentration profiles. From the graph it is observed that, as the Schmidt number increases the concentration profile increases. This can physically be explained that, the increase in Schmidt number implies that the molecular diffusion decreases that is, an increase in the Schmidt number, increases the concentration boundary layer thickness, which in turn 
MHD Arterial Blood Flow and Mass Transfer under the Presence of Stenosis, Body Acceleration and Chemical Reaction: A Case of Magnetic Therapy

increases the concentration profiles. Figure 14 shows the effect of increasing the stenotic height on concentration profile. From the graph, we have observed that, as the stenotic height slightly increases, the concentration profiles decreases. The decrease of the concentration profile is due to the reason that the size of artery gets decreased as the stenotic height increases, thus the concentration decreases too. Similar effect is observed in figure 15 where the increase in Reynolds number reduces the concentration. Figure 18 shows the effect of increasing the Hartmann number on the skin friction. From the graph, we have observed that, as the Hartman number increases, the skin friction profiles decreases. The skin friction again varies periodically with time. This physically mean that the shear stress decreases due to the presence of magnetic field. Reduction of the shear stress in turn leads to the decrease in the skin friction coefficient. On the other hand, increasing the Reynolds number implies that the inertial force increases than the viscous force. As viscous force is smaller, skin friction gets reduced. This means that, with increase of the Reynolds number, the inertial force is more dominant than the viscous force. This lowers the skin friction. This has been shown in figure 19 .

The combined effect of stenosis, body acceleration, magnetic field and chemical reaction has further been shown in figures 22 and 23. From the graphs we clearly see that the combined effect highly reduces the velocity of the blood. However, body exercise seems to have more effect than magnetic fields and stenosis. In this regard therefore, since body exercise highly raises the blood's speed, magnetic therapy for a stenosed person will therefore be of more advantageous, not only for reducing pain but also regulating blood rheology by reducing blood's velocity. Figure 23 shows the same for concentration, from the figure we see that the combined effect of stenosis, body acceleration, magnetic field and chemical reaction for the given values of parameters, generally reduce the concentration. Thus, the magnetic therapy taken during sports for the sake of reducing pain, causes reduction of transportation of some information and macromolecules in arterial blood flow. From this study we can therefore say that magnetic therapy in sports should be done with care because if a person has stenosed artery and such therapy is applied, concentration is generally affected. Medically this implies that the transfer of atherogenic molecules such as oxygen and even low-density lipoproteins from the blood to the wall get reduced. However, increasing body exercise increases the concentration.

\section{Conclusion}

Formulation of a mathematical model and computer simulations of two-dimensional blood flow through a stenosed artery in the presence of body acceleration, magnetic fields and chemical reaction has been done. Similar with previous studies, it is observed that the presence of magnetic fields affects the flow of blood. In this study it has been shown that the presence of magnetic fields in the presence of stenosis and body acceleration again affects the flow of blood. The current study gives more insights in medical use of magnetic fields in treating various diseases related to blood rheology like hypertension. It has also been observed that body exercise increases the blood's velocity. Furthermore, the presence of chemical reaction has been shown to reduce the mass concentration. In 
Annord Mwapinga, Eunice Mureithi, James Makungu and Verdiana Masanja

this regard we suggest that for patients with heart diseases, exposing them to physical exercises should be done with care as prolonged exposure to body accelerations may cause some serious health problems such as high pulse rate or even sudden death. Therapies that involve vibrations should also be handled with care by taking into account that blood's velocity will be increased. In conclusion therefore, magnetic therapy in sports is very important, it may offer multiple benefits such as reducing pain and regulating blood flow which has been increased by body accelerations.

\section{REFERENCES}

1. S.Kumari, R.Rathee and J.Nandal, Unsteady peristaltic transport of MHD Fluid through an inclined stenosed artery with slip effect, International Journal of Applied Engineering Research, 14(8) (2019) 1881-1891.

2. D.Karthikeyan and G.Jeevitha, Heat and mass transfer on MHD two phase blood flow through a stenosed artery with permeable, Wall International Journal of Innovative Technology and Exploring Engineering, 8 (2019) 1224-1232.

3. A.R.Haghighi and N.Aliashrafi, A mathematical modeling of pulsatile blood flow through a stenosed artery under effect of a magnetic field, Journal of Mathematical Modeling, 6(2)(2018)149-164.

4. M.Sharma, R.Gaur and B.Sharma, Radiation effect on MHD blood flow through a tapered porous stenosed artery with thermal and mass diffusion, International Journal of Applied Mechanics and Engineering, 24(2) (2019) 411-423.

5. V.K.Tanwar, N.Varshney and R.Agarwal, Effect of body acceleration on pulsatile blood flow through a catheterized artery, Advances in Applied Science Research, 7(2) (2016) 155-166.

6. G.Shit, M.Roy and A.Sinha, Mathematical modelling of blood flow through a tapered overlapping stenosed artery with variable viscosity, Applied Bionics and Biomechanics, 11 (2014) 185-195.

7. C.Rajashekhar, G.Manjunatha and K.Basavarajappa, Analytical solution of FHD flow of blood through two layered model in the presence of magnetic field, Journal of Mechanical Engineering Research and Developments, 40(2) (2017) 365-370.

8. V.Mwanthi, E.Mwenda and K.K.Gachoka, Velocity profiles of unsteady blood flow through an inclined circular tube with magnetic field, Journal of Advances in Mathematics and Computer Science, 24(6) (2017) 1-10.

9. L.Parmar, S.B.Kulshreshtha and D. P. Singh, The role of magnetic field intensity in blood flow through overlapping stenosed artery: A Herschel-Bulkley fluid model, Advances in Applied Science Research, 4(6) (2013) 318-328.

10. N.Mustapha and NAmin, The unsteady power law blood flow through a multiirregular stenosed artery Malaysian Journal of Industrial and Applied Mathematics, 24(2) (2008) 187-198.

11. P.Nagarani and G.Sarojamma, Flow of a Casson fluid through a stenosed artery subject to periodic body acceleration, Proceedings of the 9th WSEAS intern.Conf. Mathematical and computational methods in science and engineering, (2007) 237244.

12. K.Das and G.Saha, Arterial MHD pulsatile flow of blood under periodic body acceleration, Bulletin of Society of Mathematicians Banja Luka, 16 (2009) 21-42. 
MHD Arterial Blood Flow and Mass Transfer under the Presence of Stenosis, Body Acceleration and Chemical Reaction: A Case of Magnetic Therapy

13. K.E.Hossain and M.M.Haque, Influence of magnetic field on chemically reactive blood flow through stenosed bifurcated arteries, AIP Publishing, 1851 (2017) 1-13.

14. M.Sharma and R.Gaur, Effect of variable viscosity on chemically reacting magnetoblood flow with heat and mass transfer, Global Journal of Pure and Applied Mathematics, 13(3) (2017) 26-35. 